\title{
NUTRITION RISK SCREENING OF CANCER OUTPATIENTS IN BRITISH COLUMBIA
}

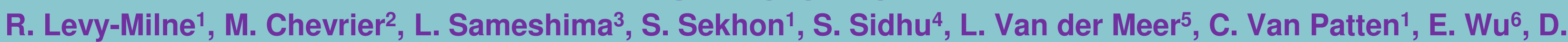
Zetterstrom $^{6}$

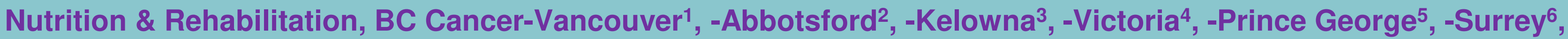
British Columbia, Canada

\section{INTRODUCTION}

The prevalence of malnutrition among cancer patients ranges from $40-80 \%$ depending on the stage, tumour site, treatments and the individual ${ }^{1}$. Malnutrition is associated with a reduced quality of life, increased risk of chemotherapy-induced toxicity, decreased response to treatment and thus, can impact disease progression and survival. Nutrition screening aims to identify patients at risk for malnutrition, ideally leading to early nutrition intervention. At BC Cancer (BCC) a Nutrition Screening Tool (NST), based on the validated Malnutrition Screening Tool (MST) $)^{2}$, is included within the self-reported outpatient intake form at the first oncologist visit.

\section{METHODS}

This study evaluated data prospectively collected from the NST of 74,797 outpatients across six regional comprehensive cancer centres in British Columbia, Canada, from 2013-2018. The data, that includes nutrition risk score, proportion of usual intake and selected symptoms, were entered into the Nutrition Screening Module of the Outcomes and Surveillance Integration System (OaSIS). Scores for malnutrition risk were based on involuntary weight loss and poor appetite as per the MST, and ranged from 0-1 (no risk), 2-3 (moderate risk) and 4-5 (high risk). Frequency distributions were estimated for each parameter on the NSTs and are presented in Figures 1 and 2.

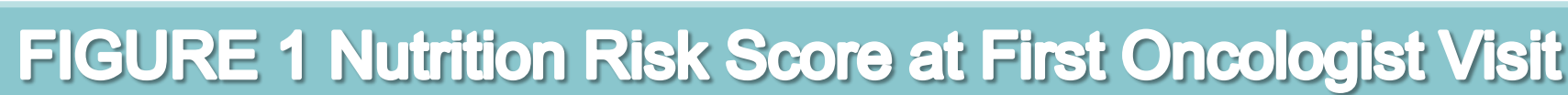

\begin{tabular}{|l|c|c|c|c|c|c|c|}
\hline Score $^{*}$ & 0 & 1 & 2 & 3 & 4 & 5 & Total \\
\hline$\#$ & 42293 & 10859 & 11339 & 6211 & 2649 & 1446 & 74797 \\
\hline$\%$ & 56.5 & 14.5 & 15.2 & 8.3 & 3.5 & 1.9 & \\
\hline
\end{tabular}

* Based on Malnutrition Screening Tool, approximately $29 \%$ scored $\geq 2$

\section{REFERENCES}

1. Dewys W.D., Begg, C., Lavin, P.T., et al. (1980). Am J Med 69:491-497.

2. 2.Ferguson, M., Capra, S., Bauer, J., \& Banks M. (1999). Nutrition 15:458-464

\section{REESULTS}

Overall, the proportion of newly admitted BCC outpatients that are at moderate to high malnutrition risk was $29.4 \%$ (Figure 1). Figure 2 shows that patients with gastrointestinal and lung cancers were at the highest risk for malnutrition whereas patients with breast and genitourinary cancers were at the lowest risk at first oncologist visit. About one-third (35\%) reported involuntarily weight loss recently. One in five patients $(22 \%)$ reported that they were eating poorly due to a decreased appetite.

FIGURE 2 Data from Nutition Sereoning at Flsst Oncologist Visit

NST Item Percentage \%

Proportion of patients scoring moderate to high malnutrition risk based on tumour site:

\begin{tabular}{r|r|} 
Gastrointestinal & 53.7 \\
Lung & 48.0 \\
Head/Neck & 27.8 \\
Genitourinary & 19.7 \\
Breast & 15.1
\end{tabular}

Involuntary weight loss

35.3 (plus 5.7\% unsure)

Poor appetite

$<50 \%$ Usual intake

Symptoms:

Diarrhea

Swallowing

\section{CONCLUSIONS}

The proportion of cancer outpatients who were at moderate to high malnutrition risk was consistent with the literature. Screening is one tool to guide triaging practice of oncology dietitians. Future research is needed to evaluate the effect of screening and repeat screening throughout the cancer care trajectory on improving patient outcomes.

\section{ACKNOWLEDGEMENTS}

We would like to thank the BCC secretaries and clerks for inputting NST data into OaSIS; Kandi Avery, Khristina Bedia, Sara Blaney, Brenda Bojsa, Jessica Campbell, Linda Douglas, Wendy MacKinnon, Maria Neale, Emily Oxley, Margaret Reeve, Stacy Rodriquez, Yvette Starchuk, Cynthia Williamson, Beverly Yhap. We would also like to thank Paul Mak for developing the Nutrition Screening Module, OaSIS. 